\title{
To Calibrate \& Validate an Agent-based Simulation Model An Application of the Combination Framework of BI Solution \& Multi-agent Platform
}

\author{
Thai Minh Truong ${ }^{1,2}$, Frédéric Amblard ${ }^{1}$, Benoit Gaudou ${ }^{1}$ and Christophe Sibertin Blanc ${ }^{1}$ \\ ${ }^{1}$ UMR 5505 CNRS-IRIT, Université Toulouse 1 Capitole, Toulouse, France \\ ${ }^{2}$ CIT, Can Tho University, Can Tho, Vietnam \\ thai.truongminh@gmail.com,\{frederic.amblard,benoit.gaudou,sibertin\}@ut-capitole.fr
}

\begin{abstract}
Keywords: Calibration, Validation, BI Solution, Data Warehouse, Multi-Agent Simulation, Agent-based Model, Brown Plant Hopper.

Abstract: Integrated environmental modeling approaches, especially the agent-based modeling one, are increasingly used in large-scale decision support systems. A major consequence of this trend is the manipulation and generation of huge amount of data in simulations, which must be efficiently managed. Furthermore, calibration and validation are also challenges for Agent-Based Modelling and Simulation (ABMS) approaches when the model has to work with integrated systems involving high volumes of input/output data. In this paper, we propose a calibration and validation approach for an agent-based model, using a Combination Framework of Business intelligence solution and Multi-agent platform (CFBM). The CFBM is a logical framework dedicated to the management of the input and output data in simulations, as well as the corresponding empirical datasets in an integrated way. The calibration and validation of Brown Plant Hopper Prediction model are presented and used throughout the paper as a case study to illustrate the way CFBM manages the data used and generated during the life-cycle of simulation and validation.
\end{abstract}

\section{INTRODUCTION}

Integrated socio-environmental modeling in general and multi-agent based simulation approach applied to socio-environmental systems in particular are increasingly used as decision-support systems in order to design, evaluate and plan public policies linked to the management of natural resources (Laniak et al., 2013). For example, in the research about invasions of Brown Plant Hopper (BPH) and the impact of $\mathrm{BPH}$ on rice fields of the Mekong Delta region (Vietnam), researchers must develop and integrate several models (e.g. BPH growth model, light-trap model, BPH migration model). They must also integrate data from different data sources and analyze the integrated data at different scales. Such an integrated simulation system involving high volume of data raises two problems: how to manage and analyze outputs of simulation models considering such a high volume of input?

Although computing power is increasing rapidly, to determine the accuracy of the simulation outputs from a large size of inputs with several parameters and to work on the high computational requirements in large systems are still the limitations of agentbased modelling (Crooks and Heppenstall, 2012). When developing a simulation model, the modelers' ambition is to achieve a credible model. To obtain a credible model or to determine the accuracy of the simulation outputs, calibration and validation are two necessary processes (Donigian, 2002; Klügl, 2008; Law, 2009). For instance, complex agentbased models are usually executed with several parameters and generate a huge amount of data, which do not have exactly the same structure than observation data from real system and can be measured and validated in various conditions. In such case, calibration and validation are used to determine which inputs and outputs are appropriate regarding observation data (Ngo and See, 2012; Rogers and Tessin, 2004; Said et al., 2002). Furthermore, calibration and validation are among the greatest challenges in agent-based modelling (Crooks et al., 2008; Crooks and Heppenstall, 2012). Therefore, how to solve the two challenges (calibration and validation) of agent-based modelling and simulation when the model deals with 
integrated systems with a high volume of input/output data?

In this article, we briefly describe a Combination Framework of BI solution and Multi-agent platform (CFBM). CFBM was designed and implemented in GAMA (Truong et al., 2013) and it can be used to model and execute agent-based simulation models, to handle data input/output of the models, and to conduct data analysis. Subsequently, we expose an approach for the calibration and validation of multiagent models by applying the CFBM, which proposes a solution to two of the limitations of agent-based modelling when working in integrated systems. In addition, a specific measure is presented, i.e. Jaccard index for ordered data sets, which has been used to evaluate the accuracy of simulation outputs.

Hence the major contribution of this article is to propose an implemented framework (CFBM) to address two crucial issues in agent-based simulation that are the calibration and validation of such models. In order to demonstrate the feasibility and the interest of the application of such framework, we apply it to the calibration and validation of a Brown Plant Hoppe invasion model, which is described briefly.

In the following sections, we first present the state of the art of works linking ABMS, BI and calibration and validation of simulation models (Section 2). The global architecture of the Combination Framework of BI solution and Multiagent platform is presented in Section 3. In Section 4 , we illustrate the calibration and validation approach for integrated agent-based simulation models. In Section 5, we apply the approach on an integrated simulation model, namely the BPH prediction model, in order to calibrate and validate the model on the GAMA simulation platform in order to illustrate the approach. Discussion and perspectives conclude this article.

\section{RELATED WORKS}

\subsection{Integration of BI Solution into a Simulation System}

Data Warehouse (DW) and analysis tools such as BI solutions can help users to manage a large amount of simulation data and to make several data analyses that support the decision-making processes (Inmon, 2005; Kimball and Ross, 2002). The combination of simulation tools and DW is on the increase and being applied in different areas. For example, although (Madeira et al., 2003; Sosnowski et al., 2007) are only two applications of OLAP technologies to a specific problem, these works demonstrate that a multidimensional database is suitable to store several hundreds of thousands of simulation results. Simulation models, DW and analysis tools with OLAP technologies were also integrated in decision support systems or forecast systems (Ehmke et al., 2011; Vasilakis et al., 2008). In (Mahboubi et al., 2010), Mahboubi et al. also used data warehouse and OLAP technologies to store and analyze a huge amount of output data generated by the coupling of complex simulation models such as biological, meteorological and so on. In particular, the authors proposed DW and Online Analytical Processing tool (OLAP tool) for storing and analyzing simulation results.

The mentioned state of the art demonstrates therefore the practical possibility and the usefulness of the combination of simulation, data warehouse and OLAP technologies. It also shows the potential of a general framework that has, as far as we know, not yet been proposed in the literature.

\subsection{Calibration and Validation}

What are calibration and validation? The calibration process is known as a test of a model with known input and output information. It is used to adjust or estimate factors for data which are not available. The validation process is the comparison of model results with numerical data independently derived from experiments or observations of the environment. These two definitions are taken from (Donigian, 2002) who was citing (ASTM, 1984). In the validation of multi-agent simulation, there are two kinds of validation: internal validation and external validation (Amblard et al., 2007). These two processes are also presented in terms of "face validation" and "statistical validation" by (Klügl, 2008). Internal validation is used to check the conformity between specifications and the implemented model. In the software engineering field, it is usually called verification and corresponds to the process which is used to compare the conceptual model to the computer-generated model. Internal validation corresponds to building the model right. External validation is used to check the similarities between the model and the real phenomenon. It is also named validation process in software engineering, so external validation corresponds to build the right model. In this paper, we address only the external validation. In the following, validation then means external validation 
of multi-agent models; and the calibration is the fine-tuning of the output of simulation model by a change in the values of parameters. The calibration involves the validation (especially the similarity evaluation which is present in both cases) to check the simulation outputs.

To calibrate and validate a simulation model, modelers used several different methods: (Donigian, 2002) used the "weight of evidence"; (Ngo and See, 2012; Rogers and Tessin, 2004; Said et al., 2002) used generic algorithms to optimize the fitness value of parameters by comparison with the observations from real systems. In general, these researchers validate simulation outputs with empirical data and check fitness conditions by statistic methods such as Root Mean Squared Error (RMSE) (Ngo and See, 2012; Willmott et al., 1985). Implementing calibration and validation model in Section 5, we tune values of parameters in their value domain (they were specified by expert biologist) by specifying the different values of all parameters and execute simulations with all possible cases (full experimental design in statistical terms). The Jaccard index (Jaccard, 1908), which can be found in (Niwattanakul et al., 2013; Rahman et al., 2010; Sachdeva et al., 2009) is used to estimate the similarity coefficient between two data sets.

\section{COMBINATION FRAMEWORK OF BUSINESS INTELLIGENCE SOLUTION AND MULTI-AGENT PLATFORM (CFBM)}

In this section, we demonstrate the logical framework to combine BI solution and Multi-agent platform. This framework has been implemented on the GAMA simulation platform (Truong et al., 2013). The CFBM is designed to handle big data from different data sources and perform analyses on the integrated data from these sources. It is a solution to improve the weaknesses of $A B M S$ when modelling is conducted on an integrated system. In this framework, we use a BI solution as a database tool, a multi-agent platform as model design tool and model execution tool. For the execution analysis tool, we can either use OLAP analysis tool or use analysis features of the platform (implemented as an external plug-in for the platform, e.g. R scripts).

The architecture of the CFBM is illustrated in Figure 1. It is formed by three systems and it supports four tools: model design tool, model execution tool, execution analysis tool and database tool.

\subsection{Simulation System}

The simulation system plays two roles: model design tool and model execution tool. It is composed of a multi-agent platform and a relational database. This system is an Online Transaction Processing (OLTP) or an operational source system. It is an outside part of the data warehouse (Kimball and Ross, 2002).

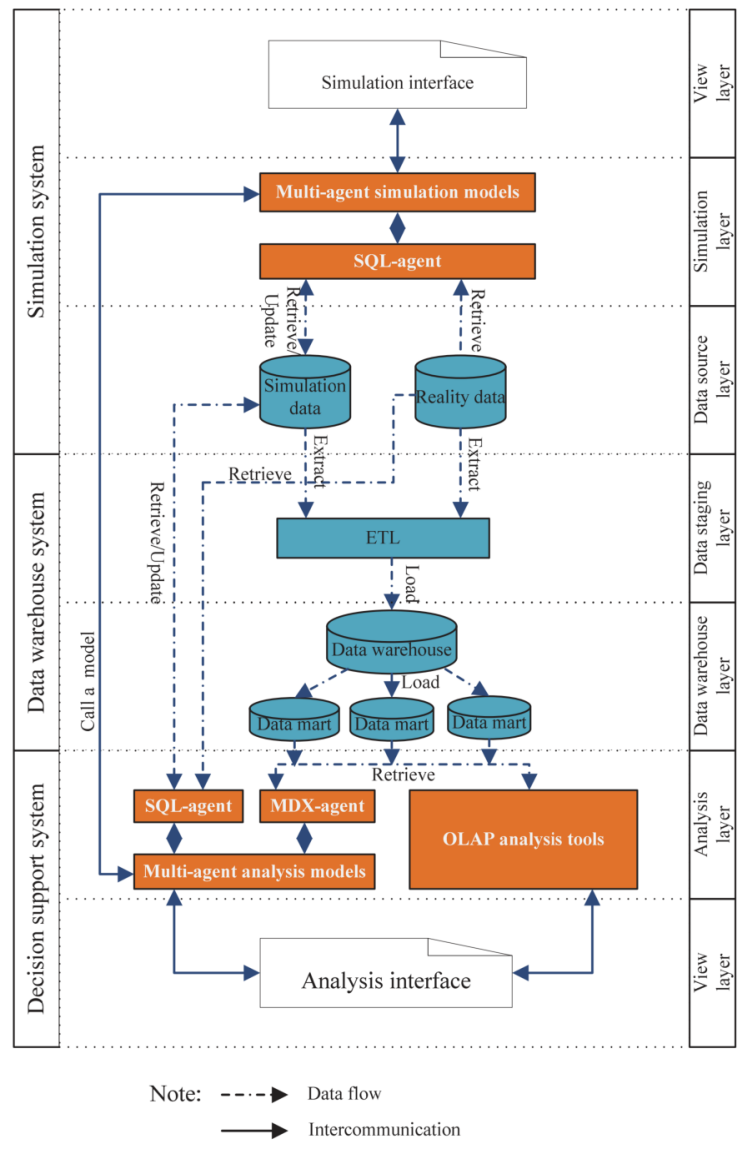

Figure 1: Combination framework of BI solution and multi-agent platform architecture.

Three layers with five components compose the simulation system. The simulation interface is the user environment that helps the modeler to design and implement his models, execute the models and visualize results. Multi-agent simulation models are a set of multi-agent based models. They are used to simulate the phenomena that the modeler aims at studying. The SQL-agent plays the role of the database tool and can access to the relational database. It is a particular kind of agent that supports Structured Query Language (SQL) functions to 
retrieve simulation inputs from simulation data or reality data, to store output simulation data into simulation data databases and to transform data (in particular the data type) from simulation model to relational database, and conversely. Reality data and Simulation data are relational databases. The reality database is used to store empirical data gathered from the target system that are needed for the simulation and analysis phases. Finally, Simulation data is used to manage simulation models, simulation scenarios and the output results of the simulation models. These two data sources will be used to feed the second part of the framework, namely the Data warehouse system

The simulation system helps to implement models, execute simulations and handle their input/output data.

\subsection{Data Warehouse System}

The data warehouse system is conceptualized as a part of the BI solution. It is an important part to integrate data from different sources (simulation data, empirical data and others external data) and is used as data storage to feed data for decision support systems.

The data warehouse system is divided into three parts. ETL (Extract-Transform-Load) is a set of processes with three responsibilities. First, it extracts all kind of data (empirical data and simulation data) from the simulation system. Second, ETL transfers the extracted data into an appropriate data format. Finally, it loads the transferred data into a data warehouse. Data warehouse is used to store historical data, which are loaded from simulation system by ETL. Data mart is a subset of data stored in the data warehouse and it is a data source for concrete analysis requirements. We can create several data marts depending on our analysis requirements. Data mart is a multidimensional database, which is designed based on multidimensional approach. It uses star joins, fact tables and dimension tables to present the data mart structure data. With a multidimensional structure, data mart is particularly useful in improving the performance of analytic processes.

\subsection{Decision Support System}

In CFBM, the decision support system plays the role of analysis tool. It is a software environment supporting analysis, decision-making features and the visualization of results. In our design, we propose to use existing OLAP analysis tools, or a multi-agent platform with analysis features or a combination of both options. The decision support system of CFBM is built on four parts. Analysis interface is a user interface used to handle analysis models and visualize results. Multi-agent analysis models are a set of agent-based analysis models. They are created based on analysis requirements and handled via analysis interface. MDX-agent is a bridge between multi-agent analysis models and data marts. This agent supports MultiDimensional eXpressions (MDX) functions to query data from a multidimensional database. OLAP analysis tools are analysis software packages that support OLAP operators.

In general, the CFBM is a solution we proposed to solve the limitations of ABMS in terms of data management and output analysis with high volume of data, which have been explained in Section 1.

The key points of the CFBM architecture are that it contains and adapts the four features of a computer simulation system (model design, model execution, execution analysis, and database management). All these functions are integrated into one multi-agent platform. The data warehouse manages the related data. The analysis models and simulation models can interact with each other. Using the CFBM architecture, we can build a simulation system not only suitable for modeling driven approach but also for data driven approaches. Furthermore, CFBM brings certain benefits for building simulation system with complex requirements such as the integration and analyzes of high volume of data.

\section{CALIBRATION \& VALIDATION APPROACH}

In this section, we propose an approach to calibrate and validate an agent-based simulation model. The approach is an application of CFBM, which we presented in Section 3. It is useful when we work with integrated simulation systems, where we need to control several models with high volume of input/output data of simulation, observation data from real system and analysis results. In this part we detail the practical use of CFBM to calibration and validation purposes.

\subsection{Calibrating an Agent-based Model}

In Figure 2, we present an automatic approach with seven steps for calibrating and validating an agentbased model. The approach helps modelers to test their models more systematically in a given 
parameter space, to evaluate (validate) outputs of each simulation and manage all data in an automatic manner.

Step 1: Load input Data with Default Parameter Values. Select a model scenario from the database then the input data and default parameter values are loaded from the quartet (model, scenario, input data set, parameters). This step assures that the correct input data and default values of parameter are loaded to simulation model.

Step 2: Execute Simulation Model. The simulation model is executed with the loaded scenario as input. In this process, outputs of the simulation are stored into a database. Because the simulation can be executed many times (replications) with the same scenario, to be sure that the system can handle results, the quartet (model, scenario, replicate, output) must be stored into the database.

Step 3: Execute Validation Model. The validation model is used to analyze the variations between simulation outputs and observations from the real system. The result of this process is a similarity coefficient or difference/distance coefficient between the output data in step 2 and observation data. The method is used to validate depends on the properties of data and on the modeler's choice. For example, we choose Jaccard index method for the validation of our model in Section 4.

In this step, the validation model loads testing data set (observations) and corresponding output data set of the quartet (model, scenario, replicate, output) to make the comparison between the two data sets. The result of validation is also stored into the database with the quartet (model, scenario, replicate, result of validation).

Step 4: Check Fitness Condition. The result of validation in Step 3 is compared with a fitness condition that is defined by the modeler. For example, the similarity coefficient of Step 3 must be greater than or equal to 0.90 (see Section 5.2.1). There are two cases:

- If the fitness is true/yes then do Step 5 (It means that the input of simulation with the value of the parameters is accepted).

- If the fitness is false/no then do Step 6 (It means that the input of simulation with the value of the parameters is not accepted).

Step 5: Store the Scenario with Fitness Parameters. Note that the result of each replication was stored in the quartet (model, scenario, replicate, result of validation) by step 3 . Hence this step only stores the adaptive scenario and fitness parameter values in the quartet (model, scenario, replicate, fitness parameter values).

Step 6: Check Adjustment Condition. The system checks if there is another instance of parameters in its population or not. Hence there are two cases:

- If the Adjustment is True/Yes then do Step 7 (It means that there is another instance of parameters in its population).

- If the Adjustment is False/No then stopping the process (It means that there is not any other instance of parameters in its population).

Step 7: Execute Adjustment Parameters. The adjustment function concerns the determination of the new values for parameters. It is used to adjust the input parameters to improve the output of the model. The result of this process is the creation of a new scenario for the simulation model.

This function changes the values of the parameters to other values in their population and progresses to step 2 .

With the seven-step approach, the calibration model can execute the simulation model with all adjusted values of the parameters, manage the whole input/output data dealt within the processes and analyze the variant between simulation outputs and observation data. It helps us to specify appropriate values of parameters automatically. The calibration model is an integration of two major models: simulation model and validation model. It also handles all data processed by the two models. The calibration model is a demonstration of the application of the CFBM, where: BI solution is used to handle all input/output of the model and empirical data related with simulating and analyzing while the analysis model is used to validate the output of the simulations.

\subsection{Validating Simulation Output using Jaccard Index}

There are several methods to measure similarity between two data sets as mentioned in (Ngo and See, 2012; Wolda, 1981). Root Mean Squared Error (RMSE) is usually used to estimate the distance (or error) between two data sets (simulation outputs and observations from real system). 


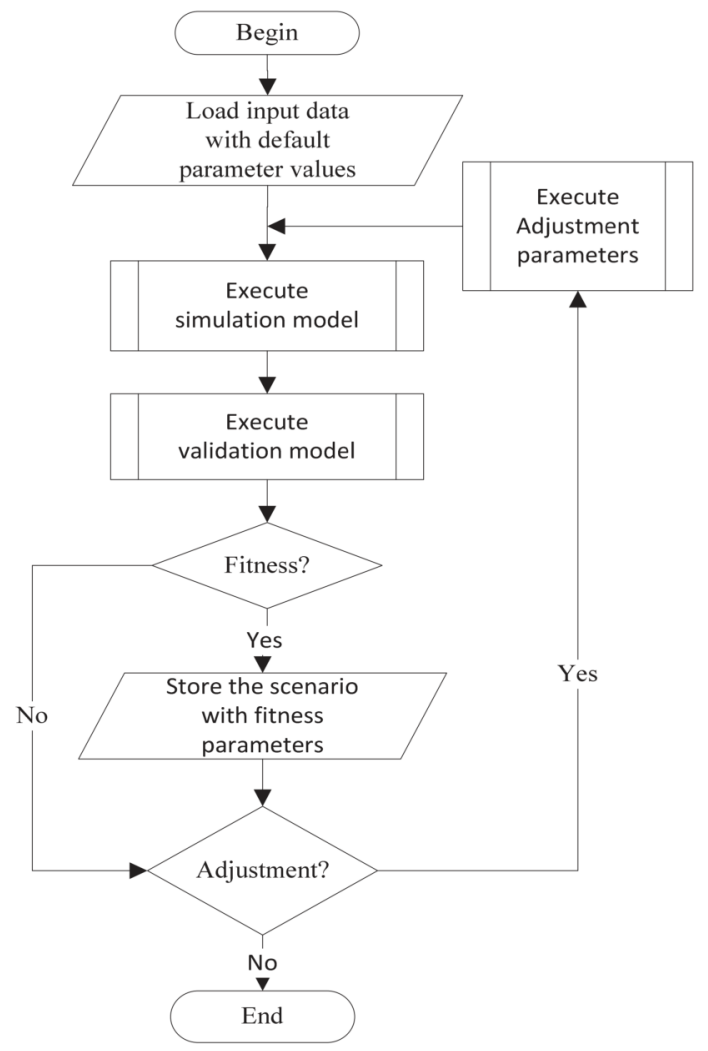

Figure 2: Workflow for the calibration of a model.

In this section, we propose a method to measure the similarity between two data sets integrating constraints on the position of elements in the data sets (ordered data set). In our method, we use Jaccard index as the similarity coefficient between two ordered data sets as follow:

Jaccard Index on Ordered Data Sets. Assume that we have two ordered data sets:

$$
\begin{aligned}
X & =\left\{x_{1}, x_{2}, \ldots, x_{n}\right\} \\
Y & =\left\{y_{1}, y_{2}, \ldots, y_{n}\right\}
\end{aligned}
$$

Definition 1: $\mathrm{x}_{\mathrm{i}}$ is called match (or similar or equal) with $y_{j}$ when $i=j$ and value of $x_{i}$ equal value of $y_{j}$. $\operatorname{match}\left(x_{i}, y_{j}\right)=\operatorname{true}$ when $i=j$ and

$$
\text { value }\left(x_{i}\right)=\operatorname{value}\left(y_{j}\right) \text { other match }\left(x_{i}, y_{j}\right)=\text { false }
$$

Definition 2: The intersection of $X$ and $Y$ is:

$$
S=\left\{s_{1}, s_{2}, \ldots, s_{n}\right\}
$$

where:

- $s_{i}=\left\{x_{i}\right\}$ (or $\left.s_{i}=\left\{y_{i}\right\}\right)$ when match $\left(x_{i}, y_{j}\right)=$ true

- $s_{i}=\{\}=\emptyset$ when $\operatorname{match}\left(x_{i}, y_{j}\right)=$ false

- $i=1$..n

Definition 3: The union of $X$ and $Y$ is:

$$
U=\left\{u_{1}, u_{2}, \ldots, u_{n}\right\}
$$

where:

- $u_{i}=\left\{x_{i}\right\}$ (or $\left.u_{i}=\left\{y_{i}\right\}\right)$ when $\operatorname{match}\left(x_{i}, y_{j}\right)=$ true

- $u_{i}=\left\{x_{i}, y_{i}\right\}$ when $\operatorname{match}\left(x_{i}, y_{j}\right)=$ false

- $i=1 . . n$

Definition 4: The cardinality of an ordered set is

$$
\begin{gathered}
|\{\}|=0 ; \\
|S|=\left|s_{1}\right|+\left|s_{2}\right|+\ldots+\left|s_{n}\right| \\
|U|=\left|u_{1}\right|+\left|u_{2}\right|+\ldots+\left|u_{n}\right|=|X|+|Y|-|S|
\end{gathered}
$$

Definition 5: Jaccard index of two ordered data sets is:

$J(X, Y)=\frac{\lfloor X \cap Y\rfloor}{\lfloor X \cup Y\rfloor}=\frac{|S|}{|U|}=\frac{c}{(a+b+c)}$

where:

- $c$ : number of matched pairs $(x i, y i)$

- $a$ : number of $x_{i}$ elements in $X$ and not matched $y_{i}$ in $Y$

- $b$ : number of $y_{i}$ elements in $Y$ and not matched $x_{i}$ in $X$

In an easier way, we calculate Jaccard index between $X$ and $Y$ based on the cardinality of $S, X$ and $Y$ as equation (6):

$J(X, Y)=\frac{\lfloor X \cap Y\rfloor}{\lfloor X \cup Y\rfloor}=\frac{|S|}{|U|}=\frac{f}{(d+e-f)}$

where:

- $f$ : cardinality of $S$.

- $d$ : cardinality of $X$.

- $e$ : cardinality of $Y$.

Example 1: Assume that we have the following data:

- Empirical data set: $X=\{1,2,3,4,5\}$

- Simulation data set: $Y=\{3,2,5,6,7\}$

Jaccard index between $X$ and $Y$ with no constraint on position of elements:

$$
\begin{gathered}
\operatorname{Intersect}(X, Y)=\{2,3,5\} \\
\operatorname{Union}(X, Y)=\{1,2,3,4,5,6,7\} \\
\mathrm{J}(X, Y) \quad=3 /(2+2+3)=3 / 7=0.429
\end{gathered}
$$

However we cannot say $\mathrm{x}_{3}=\mathrm{y}_{1}$ because we consider ordered sets. In this case, we apply Jaccard index on two ordered data sets:

$$
\text { Intersect }(X, Y)=\{\{\},\{2\},\{\},\{\},\{\}\}
$$

$\operatorname{Union}(X, Y)=\{\{1,3\},\{2\},\{3,5\},\{4,6\},\{5,7\}\}$

$(5)=>\mathrm{J}(X, Y)=1 /(4+4+1)=1 / 9=0.111$ or $(6)=>$ $\mathrm{J}(X, Y)=1 /(5+5-1)=1 / 9=0.111$

In Example 1, the similarity coefficient (Jaccard index) between two data sets with no position constraint (0.429) is different from the similarity coefficient between those two data sets with position constraint (0.111). 


\section{CALIBRATION \& VALIDATION OF THE BPH PREDICTION MODEL}

In this section, we demonstrate the calibration and validation model for an integrated agent-based simulation model, the $\mathrm{BPH}$ prediction model (Truong et al., 2013). This model is one of the research results of the DREAM ${ }^{1}$ project, coordinated between Can Tho University, Vietnam and Institut de Recherche pour le Développement (IRD), France.

\subsection{BPH Prediction Model}

BPH Prediction model is used to predict the Brown Plant Hopper (cricket) density on rice fields in Mekong Delta, Vietnam. This model contains two sub-models: BPH Growth Model and BPH Migration Model. The output is the number of BPHs in each light-trap distributed in the environment to catch BPH. Inputs and outputs of the integrated model are handled via the CFBM in GAMA. Empirical data such as administrative boundary (region, river, sea region, land used), light-trap coordinates, daily trap-densities, rice cultivated regions, general weather data (wind data), station weather data (temperature, humidity, etc.), river and sea regions are used as inputs of the simulation model and as validation data for the model.

\subsubsection{BPH Migration Model}

$\mathrm{BPH}$ migration model is used to simulate the invasion of BPHs on the rice fields. The migration process of BPHs in the studied region is modeled by a dynamical moving process on cellular automata.

Denoting $x(t)$ as the number of adult BPHs at time $t$, the migration model essentially determines the outcome $x_{\text {out }}(t)$ at a later time $t+1$ from a specific source cell and the rates of $x_{\text {out }}(t)$ moving to all destinations at time $(t+1)$. Destination cells are determined by the semi-circle under the wind, while the radius of the circle is determined by the wind velocity and the migration time in a day. The local constraints are also considered by two combinational indices: attractiveness index and obstruction index ( (Truong et al., 2013).

\footnotetext{
${ }^{1}$ http://www.ctu.edu.vn/dream/
}

\subsubsection{BPH Growth Model}

In the growth model, authors applied a deterministic model of $T$ variables where $T$ is the life cycle of the insect. To simplify the implementation process, these variables will be stored in an array variable $V$ of length $T$ where an element $V[i]$ marks the number of insects at age $i$ (i.e. $i^{\text {th }}$ day of BPH life cycle). For each simulation step, all elements of $V$ will be updated by the following equation:

$$
V[t]=\left[\begin{array}{ll}
\sum_{i \in T_{1}} V[i] * r_{b} * m, & t=1 \\
V[t-1] * r_{e n} * m, & t \in T_{2} \\
V[t-1] * r_{n a} * m, & t \in T_{3} \\
V[t-1] * m, & t \in T_{4}
\end{array}\right.
$$

where

- $\quad V[i]$ denotes the number of insects at age $i$,

- $r_{e n}$ denotes the ratio of egg number able to become the nymph,

- $r_{n a}$ denotes the ratio of nymph number able to become the adult.

- $r_{b}$ denotes the ratio of eggs can be produced by an adult.

- $m$ denotes the ratio of natural mortality.

- $T_{1}$ denotes the egg giving time span.

- $T_{2}$ denotes the egg and time span.

- $T_{3}$ denotes the nymph time span.

- $T_{4}$ denotes the adult time span.

\subsection{Calibration \& Validation of the BPH Prediction Model}

\subsubsection{Parameters for Calibration}

From equation (7) in Section 5.1.2, there are several parameters we can choose for calibration. However, we only choose $T_{4}$ (adult time span of BPH) and $m$ (the ratio of natural mortality) as two parameters for demonstration purpose. BPH has an adult time span of 8 days in minimum and 12 days in maximum. The ratio of natural mortality is 0.15 in minimum and 0.35 in maximum. The following populations of the two parameters are therefore tested:

- $T_{4}:[8,9,10,11,12]$

- $m=[0.35,0.25,0.15]$

For the input data of BPH prediction model, we used the data from 48 light-traps of three typical provinces in the Mekong Delta region: Soc Trang, Hau Giang and Bac Lieu from January 1, 2010. With one input data set, we have 15 scenarios as presented in Table 1. 
Table 1: The parameters value of scenarios for a complete experimental design.

\begin{tabular}{|c|c|c|}
\hline \multirow{2}{*}{ Scenario } & \multicolumn{2}{|c|}{ Parameters } \\
\cline { 2 - 3 } & Adult time span $\left(\boldsymbol{T}_{\mathbf{4}}\right)$ & $\begin{array}{c}\text { Ratio of natural } \\
\text { mortality }(\boldsymbol{m})\end{array}$ \\
\hline 1 & 8 & 0.35 \\
\hline 2 & 9 & 0.35 \\
\hline 3 & 10 & 0.35 \\
\hline 4 & 11 & 0.35 \\
\hline 5 & 12 & 0.35 \\
\hline 6 & 8 & 0.25 \\
\hline 7 & 9 & 0.25 \\
\hline 8 & 10 & 0.25 \\
\hline 9 & 11 & 0.25 \\
\hline 10 & 12 & 0.25 \\
\hline 11 & 8 & 0.15 \\
\hline 12 & 9 & 0.15 \\
\hline 13 & 10 & 0.15 \\
\hline 14 & 11 & 0.15 \\
\hline 15 & 12 & 0.15 \\
\hline
\end{tabular}

The Fitness Condition. We try the fitness condition in two cases:

Case 1: The difference coefficient is equal or less than 500

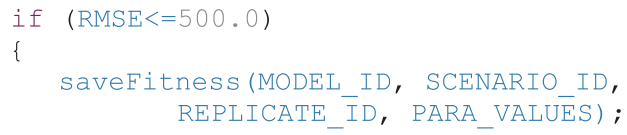

saveFitness is a user defined function, it writes the fitness scenario to database.

\subsubsection{Simulation Output and Empirical Data}

All related operations for validation in the validation model are shortly introduced in this part. As mentioned, the output of BPH prediction model is the BPH density by light-traps and by time. The empirical data (testing data) is BPH density from 48 light-traps of three typical provinces in the Mekong Delta region: Soc Trang, Hau Giang and Bac Lieu from January 1, 2010.

We simulate and predict the infection of the BPHs on the rice fields of the three provinces in 28 days. The output of the BPH prediction model has been structured as in Table 2. The structure of empirical data is presented in Table 3. Each table has 48 columns and 28 rows. The columns stand for 48 light-traps and the rows for 28 days (prediction time). In Table $2, s_{i, j}$ is the number of BPH that is simulated in step $i$ (day $i$ ) at light-trap $j$. In Table 3, $e_{i, j}$ is the number of BPHs that are caught in day $i$ at light-trap $j$ on the rice fields of Mekong Delta,
Vietnam. It should be noted that the indices of the rows and columns starts at 0 for programming reasons.

Table 2 and Table 3 present two matrixes of values which have constraints on the position (location and time) of their elements, hence they are considered as two ordered data sets.

Table 2: Simulation outputs.

\begin{tabular}{|c|c|c|c|c|}
\hline day & $\operatorname{Tr} 0$ & $\operatorname{Tr} 1$ & $\ldots$ & $\operatorname{Tr} 47$ \\
\hline 0 & $s_{0,0}$ & $s_{0,1}$ & $\ldots$ & $s_{0,47}$ \\
\hline 1 & $s_{l, 0}$ & $s_{1,1}$ & $\ldots$ & $s_{1,47}$ \\
\hline$\ldots$ & $\ldots$ & $\ldots$ & $\ldots$ & $\ldots$ \\
\hline 27 & $s_{27,0}$ & $s_{27,1}$ & $\ldots$ & $s_{27,47}$ \\
\hline
\end{tabular}

Table 3: Empirical data.

\begin{tabular}{|c|c|c|c|c|}
\hline day ${ }_{\text {dight-trap }}$ & $\operatorname{Tr} 0$ & $\operatorname{Tr} 1$ & $\ldots$ & $\operatorname{Tr} 47$ \\
\hline 0 & $e_{0,0}$ & $e_{0,1}$ & $\ldots$ & $e_{0,47}$ \\
\hline 1 & $e_{1,0}$ & $e_{1,1}$ & $\ldots$ & $e_{1,47}$ \\
\hline$\ldots$ & $\ldots$ & $\ldots$ & $\ldots$ & $\ldots$ \\
\hline 27 & $e_{27,0}$ & $e_{27,1}$ & $\ldots$ & $e_{27,47}$ \\
\hline
\end{tabular}

\subsubsection{Validating the Output of the BPH Prediction Model}

The simulation output and testing data have location constraints (light-trap) and time constraints. Hence, we use Jaccard index on ordered data sets, which has been presented in Section 4.2 to estimate the similarity between the simulation output and the empirical data. The RMSE method has also been applied to measure the difference between the two data sets.

As regard to prediction, we need to predict different periods of time: from day 0 (initial day) to $6\left(1^{\text {st }}\right.$ week $)$, from day 7 to 13 ( $2^{\text {nd }}$ week $)$, from day 14 to 20 ( $3^{\text {rd }}$ week) and from day 21 to 27 ( $4^{\text {th }}$ week). Hence for each scenario, we validate the results of simulation in four cases: $1^{\text {st }}$ week, $2^{\text {nd }}$ week, $3^{\text {rd }}$ week, $4^{\text {th }}$ week. For each case of validation, we measure difference coefficient (RMSE) and similarity coefficient (Jaccard index).

In addition, we also measure RMSE and Jaccard index of the whole data set (from day 0 to 27 or 4 weeks) for the comparison of the two measures in each scenario.

\subsubsection{The Difference Coefficient (RMSE)}

The difference coefficient between the two data sets is calculated based on the equation (8): 


$$
R M S E=\sqrt{\frac{\sum_{i=0}^{m-1} \sum_{j=0}^{n-1}\left(e_{i, j}-s_{i, j}\right)^{2}}{m * n}}
$$

where:

- $m$ denotes the number of rows of data set

- $n$ denotes the number of columns of data set

- $e_{i, j}$ is the empirical data. It denotes the number of BPHs caught in day $i$ at light-trap $j$.

- $s_{i, j}$ is the simulation output. It denotes the number of BPHs obtained in step $i$ (day $i$ ) at light-trap $j$.

The RMSE results of the 15 scenarios are presented in Table 4.

Table 4: RMSE between simulation output and empirical data.

\begin{tabular}{|c|r|r|r|r|r|}
\hline Scenario & $1^{\text {st }}$ week & $2^{\text {nd }}$ week & $3^{\text {rd }}$ week & $4^{\text {th }}$ week & 4 weeks \\
\hline 1 & $\mathbf{4 8 3 . 2 4}$ & $\mathbf{3 5 . 5 3}$ & $\mathbf{8 4 . 0 7}$ & 1610.25 & 879.58 \\
\hline 2 & $\mathbf{4 7 4 . 5 8}$ & $\mathbf{3 1 . 7 3}$ & $\mathbf{9 5 . 1 4}$ & 1714.17 & 931.34 \\
\hline 3 & $\mathbf{5 7 . 1 7}$ & $\mathbf{2 8 . 3 4}$ & $\mathbf{1 1 9 . 7 4}$ & 1762.46 & 928.09 \\
\hline 4 & $\mathbf{5 3 . 4 8}$ & $\mathbf{2 5 . 7 1}$ & $\mathbf{2 8 2 . 7 3}$ & 1768.57 & 939.68 \\
\hline 5 & $\mathbf{5 1 . 9 6}$ & $\mathbf{1 9 . 1 6}$ & $\mathbf{4 8 9 . 3}$ & 1738.06 & 944.39 \\
\hline 6 & $\mathbf{4 8 4 . 2 6}$ & $\mathbf{1 0 8 . 5 4}$ & $\mathbf{2 0 9 . 2 6}$ & 1609.61 & 885.86 \\
\hline 7 & $\mathbf{4 7 2 . 5 4}$ & $\mathbf{1 0 5 . 3 5}$ & $\mathbf{2 3 4 . 7 6}$ & 1713.14 & 937.82 \\
\hline 8 & $\mathbf{5 6 . 4 5}$ & $\mathbf{1 0 6 . 8 6}$ & $\mathbf{2 3 3 . 0 0}$ & 1761.98 & 934.39 \\
\hline 9 & $\mathbf{5 4 . 6 3}$ & $\mathbf{1 1 0 . 6 5}$ & $\mathbf{3 4 5 . 5 9}$ & 1766.14 & 945.00 \\
\hline 10 & $\mathbf{8 4 . 4 4}$ & $\mathbf{9 4 . 6 9}$ & 530.54 & 1735.76 & 950.25 \\
\hline 11 & $\mathbf{4 8 8 . 5 1}$ & 1215.93 & 2413.06 & 1863.30 & 1666.85 \\
\hline 12 & $\mathbf{4 7 3 . 8 0}$ & 1271.71 & 2639.58 & 2322.33 & 1903.59 \\
\hline 13 & $\mathbf{8 3 . 2 0}$ & 1210.19 & 2570.50 & 8826.41 & 4846.08 \\
\hline 14 & $\mathbf{1 3 9 . 4 6}$ & 1339.62 & 2636.22 & 10508.34 & 5709.14 \\
\hline 15 & 770.65 & 1150.98 & 2588.53 & 11430.14 & 6174.68 \\
\hline
\end{tabular}

Based on the difference coefficient condition $(R M S E \leq 500.0)$ in Section 5.2.1, we can show that: the RMSE of the first 14 scenarios fits the calibration conditions for the $1^{\text {st }}$ week. , this is also the case for the first 10 scenarios the $2^{\text {nd }}$ week and the first 9 scenarios the $3^{\text {rd }}$ week; but none of the 15 scenarios fits the calibration conditions of the $4^{\text {th }}$ week.

\subsubsection{The Similarity Coefficient (Jaccard Index)}

We apply equation (6) in Section 4.2 to measure the Jaccard index between the simulation output (Table 2) and empirical data (Table 3). The results are presented in Table 5.

If we compare the results in Table 5 with the similarity coefficient condition (Jindex $\geq 0.90$ ) in Section 5.2.1 then there are not any results fitting the condition. Certainly, there are no scenarios to be recognized in the calibration process. This problem can be explained by the reason that the numbers of BPHs caught at each light-trap by time has a wide range of values, from zero to ten thousands. Hence, to exactly simulate the number of BPHs at each light-trap over time is impossible or Jaccard index of two ordered data sets is not suitable to measure the similarity coefficient between two matrixes of values where the domain of elements is large. For this reason, we transformed the number of $\mathrm{BPH}$ in Table 2 and Table 3 to the BPH infection with the mapping as in Table 6. It means that we change from the ratio scale to ordinal scale. The scale in Table 6 is proposed by biologists and it was used in (Phan et al., 2010). The structures of the two transformed tables are the same as Table 2 and 3 , but the value in each cell ranges from 0 to 4 and its meaning is the BPH infection level.

Table 5: Jaccard index between simulation output and empirical data.

\begin{tabular}{|c|r|r|r|r|r|}
\hline Scenario & $1^{\text {st }}$ week & $2^{\text {nd }}$ week & $3^{\text {rd }}$ week & $4^{\text {th }}$ week & 4 weeks \\
\hline 1 & 0.4071 & 0.3352 & 0.2826 & 0.4573 & 0.3701 \\
\hline 2 & 0.4525 & 0.3035 & 0.2672 & 0.4382 & 0.3629 \\
\hline 3 & 0.4651 & 0.2586 & 0.2936 & 0.4015 & 0.3513 \\
\hline 4 & 0.4547 & 0.2553 & 0.3085 & 0.3682 & 0.3435 \\
\hline 5 & 0.3952 & 0.2785 & 0.3387 & 0.3490 & 0.3393 \\
\hline 6 & 0.1845 & 0.1482 & 0.1894 & 0.1362 & 0.1630 \\
\hline 7 & 0.1799 & 0.1516 & 0.1923 & 0.1267 & 0.1607 \\
\hline 8 & 0.1647 & 0.1320 & 0.2001 & 0.1147 & 0.1506 \\
\hline 9 & 0.1580 & 0.1922 & 0.1910 & 0.1136 & 0.1610 \\
\hline 10 & 0.1560 & 0.1804 & 0.1894 & 0.1040 & 0.1546 \\
\hline 11 & 0.1580 & 0.1239 & 0.1831 & 0.1317 & 0.1479 \\
\hline 12 & 0.1554 & 0.1430 & 0.1920 & 0.1120 & 0.1484 \\
\hline 13 & 0.1728 & 0.1371 & 0.1944 & 0.1196 & 0.1539 \\
\hline 14 & 0.1346 & 0.1701 & 0.1633 & 0.0993 & 0.1396 \\
\hline 15 & 0.1313 & 0.1649 & 0.1602 & 0.0915 & 0.1345 \\
\hline
\end{tabular}

Table 6: Transform BPH density to BPH infection.

\begin{tabular}{|c|c|l|}
\hline Number of BPH & BPH Infection & \multicolumn{1}{|c|}{ Meaning } \\
\hline$<500$ & 0 & Normal \\
\hline $50-<1500$ & 1 & Light infection \\
\hline $1500-<3000$ & 2 & Medium infection \\
\hline $3000-\leq 10000$ & 3 & Heavy infection \\
\hline$>10000$ & 4 & Hopper burn \\
\hline
\end{tabular}

We applied the Jaccard index to measure the similarity of the two transformed tables and its results are presented in Table 7.

Based on the similarity coefficient condition (Jindex $\geq 0.9$ ) in Section 5.2.1, we got the same scenarios, which are fitted with the difference coefficient conditions in Section 5.2.3.1.

From the validation results, the calibration model can choose the scenarios with parameters checking the specified fitness condition in the calibration model. 
Table 7: Jaccard index on BPH infection data sets.

\begin{tabular}{|c|r|r|r|r|r|}
\hline Scenario & $1^{\text {st }}$ week & $2^{\text {nd }}$ week & $3^{\text {rd }}$ week & $4^{\text {th }}$ week & 4 weeks \\
\hline 1 & $\mathbf{0 . 9 8 2 3}$ & $\mathbf{1 . 0 0 0 0}$ & $\mathbf{0 . 9 9 4 1}$ & 0.7819 & 0.9293 \\
\hline 2 & $\mathbf{0 . 9 8 2 3}$ & $\mathbf{1 . 0 0 0 0}$ & $\mathbf{0 . 9 8 8 2}$ & 0.7534 & 0.9187 \\
\hline 3 & $\mathbf{1 . 0 0 0 0}$ & $\mathbf{1 . 0 0 0 0}$ & $\mathbf{0 . 9 7 6 5}$ & 0.7376 & 0.9147 \\
\hline 4 & $\mathbf{1 . 0 0 0 0}$ & $\mathbf{1 . 0 0 0 0}$ & $\mathbf{0 . 9 5 3 5}$ & 0.7336 & 0.9082 \\
\hline 5 & $\mathbf{1 . 0 0 0 0}$ & $\mathbf{1 . 0 0 0 0}$ & $\mathbf{0 . 9 2 0 0}$ & 0.7376 & 0.9016 \\
\hline 6 & $\mathbf{0 . 9 8 2 3}$ & $\mathbf{0 . 9 8 6 2}$ & $\mathbf{0 . 9 5 3 5}$ & 0.7819 & 0.9169 \\
\hline 7 & $\mathbf{0 . 9 8 0 4}$ & $\mathbf{0 . 9 8 8 2}$ & $\mathbf{0 . 9 2 9 2}$ & 0.7534 & 0.9021 \\
\hline 8 & $\mathbf{1 . 0 0 0 0}$ & $\mathbf{0 . 9 8 8 2}$ & $\mathbf{0 . 9 2 7 3}$ & 0.7323 & 0.8990 \\
\hline 9 & $\mathbf{1 . 0 0 0 0}$ & $\mathbf{0 . 9 8 6 2}$ & $\mathbf{0 . 9 0 3 7}$ & 0.7297 & 0.8922 \\
\hline 10 & $\mathbf{0 . 9 9 0 1}$ & $\mathbf{0 . 9 8 6 2}$ & 0.8736 & 0.7297 & 0.8828 \\
\hline 11 & $\mathbf{0 . 9 7 6 5}$ & 0.6649 & 0.6260 & 0.4197 & 0.6396 \\
\hline 12 & $\mathbf{0 . 9 7 4 5}$ & 0.6622 & 0.6083 & 0.3989 & 0.6265 \\
\hline 13 & $\mathbf{0 . 9 8 8 2}$ & 0.6583 & 0.5975 & 0.3644 & 0.6121 \\
\hline 14 & $\mathbf{0 . 9 7 6 5}$ & 0.5975 & 0.6417 & 0.3419 & 0.5979 \\
\hline 15 & 0.8329 & 0.6970 & 0.6272 & 0.3257 & 0.5863 \\
\hline
\end{tabular}

\section{DISCUSSION}

Applying CFBM to Calibration and Validation. There have been many studies, which proposed frameworks aiming at building credible simulation models (Law, 2009) in general or at validating agent based simulation models (Klügl, 2008) in particular. Although those frameworks instructed us the processes to archive simulation model with the accuracy of the simulation output, we still need a concrete approach to solve two challenges of agentbased models, which we explained in the introductory section. By applying CFBM, we developed a calibration and validation approach for agent-based models that can help not only to handle the inputs/outputs of agent-based simulation models but also to calibrate and validate the agent-based simulation in an automatic manner. In the Section 4, we did not demonstrate the concrete method to adjust the parameters of the simulation model such as "weight of evidence" (Donigian, 2002) or generic algorithm (Ngo and See, 2012) because of two reasons: we only propose the general calibration approach and adjustment method should be implemented depending on the case study. Furthermore, our approach only concerns the management of the input/output data of simulation model and validation model and the automation of the calibration process. They are useful when working on integrated simulation systems with high amount of data. For instance, we successfully applied our approach to calibrate and validate the BPH prediction model with several data sources such as administrative boundary (region, river, sea region, land used), light-trap coordinates, daily trapdensities, rice cultivated regions, general weather data (wind data), station weather data (temperature, humidity, etc.), river and sea regions of three provinces of Mekong Delta region of Vietnam as we

explained in Section 5. It helped us to reduce time and work force.

Jaccard Index on ordered Data Sets vs. RMSE. In experiment, we also compared the Jaccard index on ordered data sets and RMSE by investigating the variation of RMSE in Table 4 and Jaccard index of the two BPH infection tables (the results of the transformation of Table 2 and 3) in Table 7. For instance, we compared the values of RMSE on the whole data sets (column 4 weeks in Table 4) with the values of Jaccard index (column 4 weeks in Table 7) by using Graphical method as Figure 3. It should be noticed that the values of RMSE in Figure 3 were divided by 1000 .

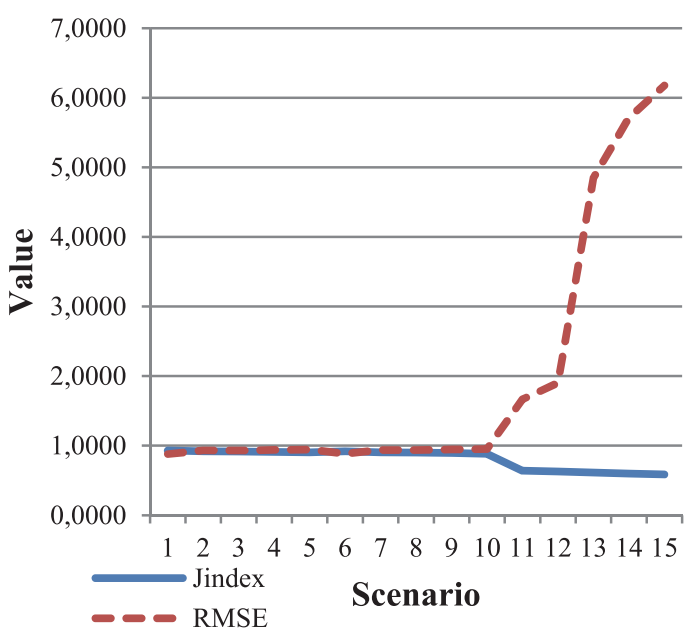

Figure 3: RMSE \& Jaccard index on whole data sets.

Figure 3 shows that there is an accordance between RMSE and Jaccard index. For instance, in scenario 1 , as the RMSE get the lowest value of 879.58, the Jaccard index obtains the highest value, which is 0.9293 . In scenario 11 , when the RMSE suddenly increases to 1666.85 , the Jaccard index decreases to 0.6396 as well. In scenario 15 , whereas RMSE gets the highest value 6174.68 , the Jaccard index gets the lowest value, i.e. 0.5863. It proves that we can use Jaccard index on ordered data for the transformed data as fitness condition, which has been presented in Section 5.2.1.

The Combination on the Fitness Condition. In the calibration of BPH prediction model, we can choose RMSE or Jaccard index as a fitness condition. However, it is suggested to use the combination of both coefficients, for instance:

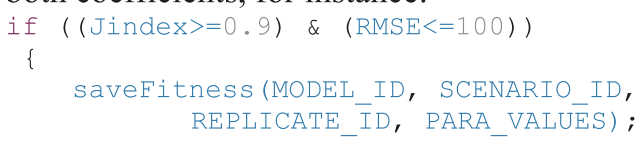


The combination of similarity and difference coefficients helps to have better fitness condition for choosing the appropriate scenario in calibration.

The Jaccard Index with Aggregation. Assume that we have two modality matrixes and the domain of the elements in $S$ and $E$ are [0..k-1], have k values:

$$
\begin{aligned}
S & =\left(\begin{array}{ccc}
s_{0,0} & \ldots & s_{0, n-1} \\
\ldots & \ldots & \ldots \\
s_{m-1,0} & \ldots & s_{m-1, n-1}
\end{array}\right) \\
E & =\left(\begin{array}{ccc}
e_{0,0} & \ldots & e_{0, n-1} \\
\ldots & \ldots & \ldots \\
e_{m-1,0} & \ldots & e_{m-1, n-1}
\end{array}\right)
\end{aligned}
$$

The aggregation on the columns on $\mathrm{S}$ (or E) has a matrix:

$C=\left(\begin{array}{ccc}c_{0,0} & \ldots & c_{0, k-1} \\ \ldots & \ldots & \ldots \\ c_{m-1,0} & \ldots & c_{m-1, k-1}\end{array}\right)$

where:

- $C$ denotes aggregation matrix on the columns of matrix $S$ (or $E$ ).

- $\quad c_{i, j}$ is the number of elements in row $i$ in $S$ (or $E$ ) having value $j$.

The aggregation on the rows on $S$ (or $E$ ) has a matrix:

$R=\left(\begin{array}{ccc}r_{0,0} & \ldots & r_{0, n-1} \\ \ldots & \ldots & \ldots \\ r_{k-1,0} & \ldots & r_{k-1, n-1}\end{array}\right)$

where:

- $R$ denotes aggregation matrix on the rows of matrix $S$ (or $E$ ).

- $\quad r_{i, j}$ is the number of elements at columns $j$ in $S$ (or $E$ ) having the value $i$.

Then we can apply the Jaccard index on ordered data sets to the aggregation matrices (equation 10,11 ) of $S$ and $E$.

\section{CONCLUSIONS}

In this paper, we introduced a conceptual framework, which is adapted to multi-agent models with high volume of data. CFBM supports experts not only to model a phenomenon and execute the models via a multi-agent platform, but also to manage a set of models with their input and output, to aggregate and analyze the model output data via data warehouse and OLAP analysis tools.

The key features of CFBM are that it supplies four components: (1) model design, (2) model execution, (3) execution analysis and (4) database management. These components are coupled and combined in a simulation system. The distinguished value of CFBM is that it augments the combination power of data warehouse, OLAP analysis tools and of a multi-agent based simulation platform. These components, when put together, are useful to develop complex simulation systems with a large amount of input/output data, which can be a what-if simulation system, a prediction/forecast system or a decision support system.

In this article, we proposed an automated calibration approach; it helps modelers to solve the limitations of ABMs concerning calibration and validation of agent-based models with high volume of data: BI solution is used to manage the high volume of input/output of the simulation models and the analysis model is used to validate the accuracy of simulation outputs on large size of input with varying parameters. We also proposed a specific method to measure the similarity coefficient of two data sets with the constraints on the position of elements, which is called "Jaccard index on the ordered data sets". In our opinion, the method can not only be used as a demonstration of validating for BPH prediction model but it is also a good approach to validate the output of other models with constraints on location and time.

Although our calibration and validation approach is the automation model with the integration of coupled models (simulation model and validation model) we have not succeeded in implementing it in GAMA. For instance, we execute the BPH prediction model with all values of the parameters via batch process. Subsequently, we execute the validation model to validate the outputs and select appropriate scenarios based on the fitness condition. These are still two separate processes but not integrated in one model as designed in Section 4. This is the problem that we plan to solve in the future.

As for further work, on one hand we will continue to develop and improve features for specific agents (SQL-agent, OLAP-agent and Analysis-agent) of the CFBM described in Section 3 to GAMA platform. On the other hand, we will also apply CFBM on multi-scale in multi-agent simulation or building what-if system, prediction system and decision support system.

\section{REFERENCES}

Amblard, F., Bommel, P., Rouchier, J., 2007. Assessment and Validation of Multi-agent Models. In: Phan, D., 
Amblard, F. (Eds.), Agent-Based Modelling and Simulation in The Social and Human Sciences. The Bardwell Press, Oxford, pp. 93-114.

ASTM, 1984. Standard Practice for Evaluating Environmental Fate Models of Chemicals. American Society of Testing Materials. Philadelphia.

Crooks, A., Castle, C., Batty, M., 2008. Key challenges in agent-based modelling for geo-spatial simulation. Comput. Environ. Urban Syst. 32, 417-430.

Crooks, A. T., Heppenstall, A. J., 2012. Introduction to Agent-Based Modeling. In: Heppenstall, A.J., Crooks, A. T., See, L. M., Batty, M. (Eds.), Agent-Based Models of Geographical Systems. Springer Netherlands, Dordrecht, pp. 85-105.

Donigian, A. S., 2002. Watershed model calibration and validation: The HSPF experience. In: Water Environment Federation. pp. 44-73.

Ehmke, J. F., Grosshans, D., Mattfeld, D. C., Smith, L. D., 2011. Interactive analysis of discrete-event logistics systems with support of a data warehouse. Comput. Ind. 62, 578-586.

Inmon, W. H., 2005. Building the Data Warehouse, 4th ed. Wiley Publishing Inc.

Jaccard, P., 1908. Nouvelles recherches sur la distribution florale. Bull Soc. Vaud. Sci. Nat 223-270.

Kimball, R., Ross, M., 2002. The Data Warehouse Toolkit: The Complete Guide to Dimensional Modeling, 2nd ed. John Wiley \& Sons, Inc.

Klügl, F., 2008. A validation methodology for agent-based simulations. In: Proceedings of the 2008 ACM Symposium on Applied Computing. pp. 39-43.

Laniak, G. F., Rizzoli, A. E., Voinov, A., 2013. Thematic issue on the future of integrated modeling science and technology. Environ. Model. Softw. 39, 13-23.

Law, A. M., 2009. How to build valid and credible simulation models. In: Simulation Conference (WSC), Proceedings of the 2009 Winter. IEEE, pp. 24-33.

Madeira, H., Costa, J. P., Vieira, M., 2003. The OLAP and data warehousing approaches for analysis and sharing of results from dependability evaluation experiments. In: International Conference on Dependable Systems and Networks. pp. 86-99.

Mahboubi, H., Faure, T., Bimonte, S., Deffuant, G., Chanet, J. P., Pinet, F., 2010. A Multidimensional Model for Data Warehouses of Simulation Results. Int. J. Agric. Environ. Inf. Syst. 1, 1-19.

Ngo, T. A., See, L., 2012. Calibration and Validation of Agent-Based Models of Land Cover Change. In: Heppenstall, A. J., Crooks, A. T., See, L. M., Batty, M. (Eds.), Agent-Based Models of Geographical Systems. Springer Netherlands, pp. 181-197.

Niwattanakul, S., Singthongchai, J., Naenudorn, E., Wanapu, S., 2013. Using of Jaccard Coefficient for Keywords Similarity. In: International MultiConference of Engineers and Computer Scientists. pp. 380-384.

Phan, C. H., Huynh, H. X., Drogoul, A., 2010. An agentbased approach to the simulation of Brown Plant Hopper (BPH) invasions in the Mekong Delta. In: Computing and Communication Technologies,
Research, Innovation, and Vision for the Future (RIVF), 2010 IEEE RIVF International Conference. IEEE, pp. 1-6.

Rahman, M., Hassan, M. R., Buyya, R., 2010. Jaccard Index based availability prediction in enterprise grids. In: Procedia Computer Science. Elsevier, pp. 2707-2716.

Rogers, A., Tessin, P. von, 2004. Multi-objective calibration for agent-based models.

Sachdeva, V., Freimuth, D., Mueller, C., 2009. Evaluating the jaccard-tanimoto index on multi-core architectures. In: Computational Science-ICCS 2009. Springer Berlin Heidelberg, pp. 944-953.

Said, L. B., Bouron, T., Drogoul, A., 2002. Agent-based interaction analysis of consumer behavior. In: The First International Joint Conference on Autonomous Agents and Multiagent Systems: Part 1. ACM. pp. 184-190.

Sosnowski, J., Zygulski, P., Gawkowski, P., 2007. Developing Data Warehouse for Simulation Experiments. In: Rough Sets and Intelligent Systems Paradigms. Springer Berlin Heidelberg, pp. 543-552.

Truong, T. M., Truong, V. X., Amblard, F., Drogoul, A., Benoit, G., Huynh, H. X., Le, M. N., Sibertin-blanc, C., 2013. An implementation of framework of Business Intelligence for Agent-based Simulation. In: The 4th International Symposium on Information and Communication Technology (SoICT 2013).

Truong, V. X., Huynh, H. X., Le, M. N., Drogoul, A., 2013. Optimizing an Environmental Surveillance Network with Gaussian Process - An optimization approach by agent-based simulation. In: The Sixth International KES Conference on Agents and MultiAgent Systems - Technologies and Applications (KES AMSTA 2013). IOS Press, pp. 102-111.

Vasilakis, C., El-Darzi, E., Chountas, P., 2008. A decision support system for measuring and modelling the multiphase nature of patient flow in hospitals. In: Intelligent Techniques and Tools for Novel System Architectures. Springer Berlin Heidelberg, pp. 201-217.

Willmott, C. J., Ackleson, S. G., Davis, R. E., Feddema, J. J., Klink, K. M., Legates, D. R., O’Donnell, J., Rowe, C. M., 1985. Statistics for the evaluation and comparison of models. J. Geophys. Res. 90, 89959005.

Wolda, H., 1981. Similarity indices, sample size and diversity. Oecologia 50.3, 296-302. 